\title{
Domain Decomposition Preconditioning for High Order Hybrid Discontinuous Galerkin Methods on Tetrahedral Meshes
}

\author{
Joachim Schöberl and Christoph Lehrenfeld
}

Bericht Nr. 335

Februar 2012

Key words: Hybrid Discontinuous Galerkin methods, $\mathrm{p}$-version finite elements, domain decomposition

AMS subject classifications: $65 \mathrm{~N} 30,65 \mathrm{~F} 08$

Institut für Geometrie und Praktische Mathematik RWTH Aachen

Templergraben 55, D-52056 Aachen (Germany) 


\title{
Domain Decomposition Preconditioning for High Order Hybrid Discontinuous Galerkin Methods on Tetrahedral Meshes
}

\author{
Joachim Schöberl and Christoph Lehrenfeld
}

February 7, 2012

\begin{abstract}
Hybrid discontinuos Galerkin methods are popular discretization methods in applications from fluid dynamics and many others. Often large scale linear systems arising from elliptic operators have to be solved. We show that standard $p$-version domain decomposition techniques can be applied, but we have to develop new technical tools to prove poly-logarithmic condition number estimates, in particular on tetrahedral meshes.
\end{abstract}

\section{Introduction}

In this paper we are concerned with discontinuous Galerkin (DG) finite element methods for elliptic problems $[4,12,23]$. The motivation might be to have dominant convection, or one wants to build exactly divergence free finite element spaces for incompressible flows [11,30], or other. We think of operator splitting methods, where one has to solve a large scale symmetric matrix equation in each time-step.

In recent years hybridization methods appeard, which allow to reduce the discrete system to the element interfaces [10]. This paper is concerned with the construction and analysis of domain decomposition methods for the Hybrid Discontinuous Galerkin (HDG) method. We consider one element as sub-domain, and the coarse grid problem consists of mean values on element interfaces. We prove robustness with respect to the mesh-size, and a poly-logarithmic growth of the condition number with the polynomial order $p$.

There is now an established literature on high order finite element methods, from the more theoretical point of view as well as from an applied one [41, 39, 25, 13].

We consider two strategies for domain decomposition algorithms [43], non-overlapping Schwarz type methods $[15,19,20]$ and balancing domain decomposition with constraints (BDDC) $[14,31]$. There is a big literature, in particular high order methods and three dimensional problems are treated in $[2,5,7,8,9,18,22,26,27,29,29,34,35,36,38,40]$. There is a classical paper on multi-level analysis for h-version DG methods by Gopalakrishnan and Kanschat [17], and a recent one studying higher order methods by Antonietti and Houston [3] showing a polynomial growth of the condition number in $p$. We will see that the conditioning is significantly improved by hybridization, namely to a poly-logarithmic growth. We are not aware of particular analysis for preconditioners for high order HDG methods, even not in 2D.

The main result of the present paper is Theorem 3 proving that optimal extension from faces to elements with Dirichlet constraints is nearly as good as extension without constraints. With this result condition number estimates follow with the usual techniques. 
The main difficulty is to build optimal extension operators from an edge to a tetrahedron. This problem was solved for hexahedal elements by multiplying with fast decaying functions by Pavarino and Widlund [36]. Polynomial extension operators for simplicial elements are usually based on smoothing operators [5, 32]. Heuer and Leydecker have analyzed such operators also for boundary elements, i.e, for three dimensional edge to face extension.

We cannot use the existing simplicial extension operators to prove quasi-optimality of HDG methods since they do not decay fast enough in the jump-norm. We give a new construction of discrete edge-to-tetrahedron extension operators which are motivated by the multiplication with low-energy functions of Pavarino and Widlund, but are contained in the polynomial space on tetrahedra.

We declare some notation. With $a \preceq b$ we mean the existence of a generic constant $c$ such that $a \leq c b$, where $c$ is independet of parameters $h$ and $p$. Otherwise, we denote the dependence as $c(p)$. The space of univariate polynomials of order $p$ is $P^{p}$, and $P^{p}(T)$ is the space of multivariate polynomials of total order $p$ on a simplex $T$. To simplify notation we redefine $\log p:=1$ for $p \in\{0,1\}$.

In Section 2 we give the hybrid DG formulation, in Section 3 we prove the main result, Theorem 3, and show how to apply it to analyze domain decomposition algorithms for HDG. Technical lemmas are shifed to Sections 4, 5, and 6. In Section 4 we collect properties of orthogonal polynomials, and prove one dimensional trace estimates and construct onedimensional extension operators with respect to different norms. The short Section 5 gives the proofs for extension from vertices, the main technical proves for the extension from edges are in Section 6.

\section{HDG discretization}

Let $\Omega \subset \mathbb{R}^{3}$ be a polyhedral domain. Let $\mathcal{T}=\{T\}$ be a conforming triangulation of $\Omega$ consisting of shape regular tetrahedral elements. With $\mathcal{F}=\{F\}$ we denote the set of all faces, and $\mathcal{F}_{T}$ are the faces of the element $T$. As usual $h_{T}=\operatorname{diam} T$ is the local mesh-size.

We consider the Dirichlet problem of the Poisson equation problem, namely

$$
-\Delta u=f \text { in } \Omega, \quad u=0 \text { on } \partial \Omega,
$$

with the source $f \in L_{2}(\Omega)$. We define the $p^{\text {th }}$ order hybrid discontinuous Galerkin finite element space

$$
V_{N}:=P^{p}(\mathcal{T}) \times P^{p}(\mathcal{F}):=\prod_{T \in \mathcal{T}} P^{p}(T) \times \prod_{F \in \mathcal{F}} P^{p}(F),
$$

its subspace $V_{N, 0}=\left\{(u, \lambda) \in V_{N}: \lambda=0\right.$ on $\left.\partial \Omega\right\}$, and the hybrid discontinuous Galerkin (HDG) method as: find $(u, \lambda) \in V_{N, 0}$ :

$$
A(u, \lambda ; v, \mu)=(f, v)_{L_{2}(\Omega)} \quad \forall(v, \mu) \in V_{N, 0} .
$$

The HDG bilinear-form is

$$
A(u, \lambda ; v, \mu)=\sum_{T \in \mathcal{T}} A_{T}(u, \lambda ; v, \mu)
$$

with the element contributions

$$
A_{T}(u, \lambda ; v, \mu):=\int_{T} \nabla u \nabla v+\int_{\partial_{T}} \frac{\partial u}{\partial n}(\mu-v)+\int_{\partial T} \frac{\partial v}{\partial n}(\lambda-u)+\alpha(u-\lambda, v-\mu)_{j, \partial T}
$$


with a fixed $\alpha>4=\left|\mathcal{F}_{T}\right|$. We choose the stabilization similar to the stabilized Bassi-Rebay method $[6,4,24]$ as

$$
(u-\lambda, v-\mu)_{j, \partial T}=\sum_{F \in \mathcal{F}_{T}}\left(r_{F}(u-\lambda), r_{F}(v-\mu)\right)_{L_{2}(T)} .
$$

The discrete lifting operator $r_{F}: P^{p}(F) \rightarrow\left[P^{p}(T)\right]^{3}$ is defined by

$$
\left(r_{F}(\mu), v\right)_{L_{2}(T)}=(\mu, v \cdot n)_{L_{2}(F)} \quad \forall v \in\left[P^{p}(T)\right]^{3} .
$$

The norm

$$
\|u-\lambda\|_{j, F}=\left\|r_{F}(u-\lambda)\right\|_{L_{2}(T)}
$$

is realized by

$$
\|u-\lambda\|_{j, F}=\sup _{\sigma \in\left[P^{p}(T)\right]^{3}} \frac{(u-\lambda, \sigma \cdot n)_{L_{2}(F)}}{\|\sigma\|_{L_{2}(T)}}=\sup _{\sigma \in P^{p}(T)} \frac{(u-\lambda, \sigma)_{L_{2}(F)}}{\|\sigma\|_{L_{2}(T)}} .
$$

The last equality holds since the normal vector $n$ is constant on $F$.

We define the norm

$$
\|(u, \lambda)\|_{1, H D G}^{2}:=\sum_{T \in \mathcal{T}}\left\{\|\nabla u\|_{L_{2}(T)}^{2}+\|u-\lambda\|_{j, \partial T}^{2}\right\}
$$

with $\|u-\lambda\|_{j, \partial T}^{2}=\sum_{F \in \mathcal{F}_{T}}\|u-\lambda\|_{j, F}^{2}$. We note that more general elliptic equations, with mixed boundary conditions, variable coefficients as well as variable polynomial orders can be treated the same way.

Theorem 1. The HDG bilinear-form $A(.,$.$) is continuous and coercive on \left(V_{N, 0},\|\cdot\|_{1, H D G}\right)$.

Proof. Continuity and coercivity are proven element-wise, i.e.,

$$
\|\nabla u\|_{L_{2}(T)}^{2}+\|u-\lambda\|_{j, \partial T}^{2} \preceq A_{T}(u, \lambda ; u, \lambda) \preceq\|\nabla u\|_{L_{2}(T)}^{2}+\|u-\lambda\|_{j, \partial T}^{2}
$$

is shown for all $u \in P^{p}(T), \lambda \in P^{p}\left(\mathcal{F}_{T}\right)$, and for all $T \in \mathcal{T}$. For $F \in \mathcal{F}_{T}$ we use Young's inequality $a b \leq \frac{1}{2 \gamma} a^{2}+\frac{\gamma}{2} b^{2}$ with $4<\gamma<\alpha$ to obtain

$$
\begin{aligned}
\int_{F} \frac{\partial u}{\partial n}(u-\lambda) & \leq\|\nabla u\|_{L_{2}(T)} \sup _{\sigma \in\left[P^{p}\right]^{3}} \frac{\int \sigma_{n}(u-\lambda)}{\|\sigma\|_{L_{2}(T)}} \\
& \leq \frac{1}{2 \gamma}\|\nabla u\|_{L_{2}(T)}^{2}+\frac{\gamma}{2}\|u-\lambda\|_{j, F}^{2} .
\end{aligned}
$$

Summing over the 4 faces of $T$ we obtain

$$
\begin{aligned}
A_{T}(u, \lambda ; u, \lambda) & =\|\nabla u\|_{L_{2}(T)}^{2}+2 \sum_{F \in \mathcal{F}_{T}} \int_{F} \frac{\partial u}{\partial n}(u-\lambda)+\alpha(u-\lambda, u-\lambda)_{j, T} \\
& \geq\|\nabla u\|_{L_{2}(T)}^{2}-\frac{4}{\gamma}\|\nabla u\|_{L_{2}(T)}^{2}-\gamma \sum_{F}\|u-\lambda\|_{j, F}^{2}+\alpha\|u-\lambda\|_{j, \partial T}^{2} \\
& \succeq\|\nabla u\|_{L_{2}(T)}^{2}+\|u-\lambda\|_{j, \partial T}^{2},
\end{aligned}
$$

continuity is verified similar. 
Theorem 2. For $F \in \mathcal{F}_{T}$ let $\mathbf{P}^{k}$ denote the $L_{2}(F)$-orthogonal projector onto $P^{k}(F)$, with $\mathbf{P}^{-1}=0$. For $\lambda \in P^{p}(F)$ there holds

$$
\|\lambda\|_{j, F}^{2} \simeq h_{T}^{-1} \sum_{k=0}^{p} p(p-k+1)\left\|\left(\mathbf{P}^{k}-\mathbf{P}^{k-1}\right) \lambda\right\|_{L_{2}(F)}^{2}
$$

Proof. By an affine-linear transformation to the reference tetrahedron and reference face

$$
\begin{aligned}
& T=\{(x, y, z): y \geq 0, z \geq 0,|x|+y+z \leq 1\} \\
& F=\{(x, y, 0): y \geq 0,|x|+y \leq 1\}
\end{aligned}
$$

one obtains the scaling in the mesh-size. By means of Jacobi and Legendre polynomials (see Section 4), the Dubiner basis polynomials $[16,25]$

$$
\varphi_{i j}(x, y)=P_{i}\left(\frac{x}{1-y}\right)(1-y)^{i} P^{(0,2 i+1)}(1-2 y) \quad \text { for } i+j \leq p
$$

form an $L_{2}(F)$-orthogonal basis for $P^{p}(F)$. Expand

$$
\begin{aligned}
\lambda(x, y) & =\sum_{i+j \leq p} \lambda_{i j} \varphi_{i j}(x, y) \\
\sigma(x, y, z) & =\sum_{i+j \leq p} \varphi_{i j}\left(\frac{x}{1-z}, \frac{y}{1-z}\right)(1-z)^{i+j} \sigma_{i j}(z)
\end{aligned}
$$

with $\sigma_{i j} \in P^{p-i-j}$. By the change of variables

$$
g: F \times[0,1] \rightarrow T:(\xi, \eta, z) \mapsto(x, y, z):=((1-z) \xi,(1-z) \eta, z)
$$

with $\operatorname{det} g^{\prime}=(1-z)^{2}$ we express

$$
\|\sigma\|_{L_{2}(T)}^{2}=\int_{F} \int_{0}^{1}(1-z)^{2} \sigma((1-z) \xi,(1-z) \eta, z)^{2} d z d(\xi, \eta) .
$$

Due to orthogonality there holds

$$
\|\sigma\|_{L_{2}(T)}^{2}=\sum_{i+j \leq p}\left\|\varphi_{i j}\right\|_{L_{2}(F)}^{2} \int_{0}^{1}(1-z)^{2 i+2 j+2} \sigma_{i j}^{2}(z) d z
$$

and

$$
(\lambda, \sigma)_{L_{2}(F)}=\sum_{i+j \leq p}\left\|\varphi_{i j}\right\|_{L_{2}(F)}^{2} \lambda_{i j} \sigma_{i j}(0)
$$

There holds

$$
\sup _{\sigma \in P^{p}(T)} \frac{(\lambda, \sigma)_{L_{2}(F)}^{2}}{\|\sigma\|_{L_{2}(T)}^{2}}=\left\|\sigma^{*}\right\|_{L_{2}(T)}^{2}
$$

where $\sigma^{*} \in P^{p}(T)$ solves

$$
\left(\sigma^{*}, \tau\right)_{L_{2}(T)}=(\lambda, \tau)_{L_{2}(F)} \quad \forall \tau \in P^{p}(T) .
$$


The components $\sigma_{i j}^{*} \in P^{p-i-j}$ of the $L_{2}(T)$-orthogonal decomposition

$$
\sigma^{*}(x, y, z)=\sum_{i+j \leq p} \varphi_{i j}\left(\frac{x}{1-z}, \frac{y}{1-z}\right)(1-z)^{i+j} \sigma_{i j}^{*}(z)
$$

solve

$$
\int_{0}^{1}(1-z)^{2 i+2 j+2} \sigma_{i j}^{*}(z) \tau(z) d z=\lambda_{i j} \tau(0) \quad \forall \tau \in P^{p-i-j},
$$

and there holds

$$
\int_{0}^{1}(1-z)^{2 i+2 j+2} \sigma_{i j}^{*}(z)^{2} d z=\sup _{\sigma_{i j} \in P^{p-i-j}} \frac{\left(\lambda_{i j} \sigma_{i j}(0)\right)^{2}}{\int_{0}^{1}(1-z)^{2 i+2 j+2} \sigma_{i j}^{2}(z) d z} .
$$

From Lemma 6 below we get

$$
\left|\sigma_{i j}(0)\right|^{2} \preceq p(p-i-j+1) \int_{0}^{1}(1-z)^{2 i+2 j+2} \sigma_{i j}^{2}(z) d z
$$

is sharp, and thus

$$
\int_{0}^{1}(1-z)^{2 i+2 j+2} \sigma_{i j}^{*}(z)^{2} d z \simeq p(p-i-j+1) \lambda_{i j}^{2}
$$

Thus there holds

$$
\begin{aligned}
\sup _{\sigma \in P^{p}(T)} \frac{(\lambda, \sigma)_{L_{2}(F)}^{2}}{\|\sigma\|_{L_{2}(T)}^{2}} & \simeq \sum_{i+j \leq p} p(p-i-j+1) \lambda_{i j}^{2}\left\|\varphi_{i j}\right\|_{L_{2}(F)}^{2} \\
& =\sum_{k=0}^{p} p(p-k+1) \sum_{i+j=k} \lambda_{i j}^{2}\left\|\varphi_{i j}\right\|^{2} \\
& =\sum_{k=0}^{p} p(p-k+1)\left\|\left(\mathbf{P}^{k}-\mathbf{P}^{k-1}\right) \lambda\right\|_{L_{2}(F)}^{2} .
\end{aligned}
$$

We observe that

$$
\frac{p}{h}\|u-\lambda\|_{L_{2}(F)}^{2} \preceq\|u-\lambda\|_{j, F}^{2} \preceq \frac{p^{2}}{h}\|u-\lambda\|_{L_{2}(F)}^{2} .
$$

Often $\frac{\alpha p^{2}}{h}\|u-\lambda\|_{L_{2}(F)}^{2}$ with a sufficiently large parameter $\alpha$ is chosen as penalty term. Usually $\alpha$ is chosen on the safe side. We will see in the numerical examples that the condition number does increase with $\alpha$. In this paper we prove quasi-optimal condition numbers for the presented stabilization, it does not carry over to the weighted $L_{2}$-stabilization.

The benefit is two-fold, on one side the method is guaranteed to be stable, for any $\alpha>\left|\mathbf{F}_{T}\right|$, on the other side the condition number is proven to have only poly-logarithmic growth. 


\section{Domain decomposition preconditioning}

The analysis of non-overlapping DD preconditioners is based on stable decompositions of finite element functions. For that, quasi-optimal extension procedures are essential. The main result of our work is to construct an extension operator, and bound its norm.

For $F \in \mathcal{F}$ and a fixed $T \in \mathcal{T}$ such that $F \subset T$ we define the trace semi-norm

$$
\|\lambda\|_{F}^{2}=\inf _{u \in P^{p}(T)}\left\{\|\nabla u\|_{L_{2}(T)}^{2}+\|u-\lambda\|_{j, F}^{2}\right\}
$$

and the trace norm

$$
\|\lambda\|_{F, 0}^{2}=\inf _{u \in P^{p}(T)}\left\{\|\nabla u\|_{L_{2}(T)}^{2}+\|u-\lambda\|_{j, F}^{2}+\sum_{\substack{F^{\prime} \in \mathcal{F}_{T} \\ F^{\prime} \neq F}}\|u-0\|_{j, F^{\prime}}^{2}\right\} .
$$

The semi-norm $\|\lambda\|_{F}$ mimics the $H^{1 / 2}(F)$ semi-norm, i.e. the trace semi-norm corresponding to arbitrary $H^{1}$-optimal extension onto the element $T$, while the norm $\|\lambda\|_{F, 0}$ mimics the $H_{00}^{1 / 2}$-norm, i.e., the trace norm corresponding to $H^{1}$-optimal extension under Dirichlet constraints on $\partial T \backslash F$. Note that for continuous finite element spaces $\|\lambda\|_{H_{00}^{1 / 2}}$ is defined only for $\lambda=0$ on $\partial F$. For hybrid DG, both norms $\|\lambda\|_{F}$ and $\|\lambda\|_{F, 0}$ are defined for the same space $P^{p}(F)$.

Theorem 3. Let $\lambda_{F} \in P^{p}(F)$ with $\int_{F} \lambda=0$. Then here holds

$$
\|\lambda\|_{F, 0}^{2} \preceq(\log p)^{\gamma}\|\lambda\|_{F}^{2}
$$

with $\gamma=3$.

Proof. It is enough to consider the reference element $T$. Let $u$ be the minimizer corresponding to $\|\lambda\|_{F}$. Thanks to a Poincare - type inequality and Theorem 2 there holds

$$
\begin{aligned}
\|u\|_{H^{1}(T)} & \preceq\|\nabla u\|_{L_{2}(T)}^{2}+\left(\int_{F} u\right)^{2} \\
& \preceq\|\nabla u\|_{L_{2}(T)}^{2}+\left(\int_{F} u-\lambda\right)^{2}+\left(\int_{F} \lambda\right)^{2} \\
& \preceq\|\nabla u\|_{L_{2}(T)}^{2}+\|u-\lambda\|_{j, F}^{2} .
\end{aligned}
$$

We modify the function $u$ by subtracting vertex and edge contributions:

$$
\begin{aligned}
& u_{2}=u-\sum_{V \subset F} \mathcal{E}_{V \rightarrow T} u(V) \\
& u_{3}=u_{2}-\left.\sum_{E \subset F} \mathcal{E}_{E \rightarrow T} u_{2}\right|_{E}
\end{aligned}
$$

In Theorem 11 and Theorem 18 below we prove that the function $u_{3}$ is in $P^{p}$, vanishes on $\partial F$, and satisfies

$$
\left\|\nabla u_{3}\right\|_{L_{2}(T)}^{2}+\left\|u-u_{3}\right\|_{j, F}^{2} \preceq \log p\|u\|_{H^{1}(T)}^{2} .
$$

There holds $\left\|u_{3}\right\|_{H_{00}^{1 / 2}(F)}^{2} \preceq(\log p)^{2}\left\|u_{3}\right\|_{H^{1 / 2}(F)}^{2}[8]$, Lemma 4.7. Now take

$$
\tilde{u}:=\left.\mathcal{E}_{F \rightarrow T} u_{3}\right|_{F}
$$


as the Muñoz-Sola extension [32]. Finally we get

$$
\|\nabla \tilde{u}\|_{L_{2}(T)}^{2}+\|\tilde{u}-\lambda\|_{j, F}^{2} \preceq(\log p)^{\gamma}\|\lambda\|_{F}^{2},
$$

and together with $\tilde{u}=0$ on $\partial T \backslash F$ we have proven the result.

We note that in $[36,8,35]$ and others estimates with $(\log p)^{2}$ have been obtained for continuous finite elements. It might be that our result can also be improved to $(\log p)^{2}$. One approach would be to directly estimate the $\int_{F} \frac{1}{\operatorname{dist}(x, \partial F)} u(x)^{2} d x$ term of the $H_{00}^{1 / 2}(F)$-norm. If one succeeds with that estimate, then that improved $\gamma$ can be used immediately in the following condition number estimates.

\subsection{Schwarz type domain decomposition}

To analyze Scharz-type domain decomposition methods one has to prove stable decompositions into sub-spaces.

For $\lambda \in P^{p}(\mathcal{F})$ we define the Schur-complement norm

$$
\|\lambda\|_{S}^{2}=\inf _{u \in P^{p}(\mathcal{T})}\|(u, \lambda)\|_{1, H D G}^{2} .
$$

Theorem 4. Let $\lambda \in P^{p}(\mathcal{F})$. Define the coarse grid component as

$$
\lambda_{H} \in P^{0}(\mathcal{F}) \text { such that } \int_{F} \lambda_{H}=\int_{F} \lambda,
$$

and for $F \in \mathcal{F}$ define the local components $\lambda_{F}$ as

$$
\lambda_{F}=\left\{\begin{array}{cl}
\lambda_{\mid F}-\lambda_{H \mid F} & \text { on } F \\
0 & \text { for } F^{\prime} \neq F
\end{array}\right.
$$

Then there holds

$$
\left\|\lambda_{H}\right\|_{S}^{2}+\sum_{F \in \mathcal{F}}\left\|\lambda_{F}\right\|_{S}^{2} \preceq(\log p)^{\gamma}\|\lambda\|_{S}^{2}
$$

Thus, the additive Schwarz preconditioner $C_{A S M}$ applied to the facet Schur-complement $S_{A}$ of $A$ leads to a condition number estimate

$$
\kappa\left(C_{A S M}^{-1} S_{A}\right) \preceq(\log p)^{\gamma}
$$

Proof. From the definitions of the norms there follows

$$
\sum_{F \in \mathcal{F}}\left\|\left.\mu\right|_{F}\right\|_{F}^{2} \preceq\|\mu\|_{S}^{2} \preceq \sum_{F \in \mathcal{F}}\left\|\left.\mu\right|_{F}\right\|_{F, 0}^{2} \quad \forall \mu \in P^{p}(\mathcal{F}) .
$$

Since $\int_{F} \lambda_{F}=0$ we have

$$
\sum_{F \in \mathcal{F}}\left\|\lambda_{F}\right\|_{S}^{2} \preceq \sum_{F \in \mathcal{F}}\left\|\left.\lambda_{F}\right|_{F}\right\|_{F, 0}^{2} \preceq(\log p)^{\gamma} \sum_{F \in \mathcal{F}}\left\|\left.\lambda_{F}\right|_{F}\right\|_{F}^{2}=(\log p)^{\gamma} \sum_{F \in \mathcal{F}}\left\|\left.\lambda\right|_{F}\right\|_{F}^{2} \preceq(\log p)^{\gamma}\|\lambda\|_{S}^{2},
$$

and

$$
\left\|\lambda_{H}\right\|_{S}^{2}=\left\|\lambda-\sum_{F \in \mathcal{F}} \lambda_{F}\right\|_{S}^{2} \preceq\|\lambda\|_{S}^{2}+\left\|\sum_{F \in \mathcal{F}} \lambda_{F}\right\|_{S}^{2} \preceq\|\lambda\|_{S}^{2}+\sum_{F \in \mathcal{F}}\left\|\lambda_{F \mid F}\right\|_{F, 0}^{2} \preceq(\log p)^{\gamma}\|\lambda\|_{S}^{2} .
$$

Due to finite overlap of the sub-spaces, the the largest eigenvalue of $C_{A S M}^{-1} S$ is bounded by a constant, and thus the condition number is bounded by $(\log p)^{\gamma}$. 


\subsection{BDDC preconditioners}

To define a BDDC preconditioner one sub-divides degrees of freedom into primal and dual. The dual ones are treated discontinuous, and thus can be eliminated on the element-level. In our case we choose the mean value on the face as primal, all others are dual degrees of freedom. Thus, the remaining global system involves only one degree of freedom per face.

Theorem 5. The BDDC preconditioner with mean values on faces leads to a condition number

$$
\kappa\left(C_{B D D C}^{-1} S_{A}\right) \preceq(\log p)^{\gamma}
$$

Proof. Let $\lambda$ be double-valued on faces with consistent mean-values, this means

$$
\lambda=\left(\lambda_{T}\right)_{T \in \mathcal{T}} \in \prod_{T \in \mathcal{T}} P^{p}\left(\mathcal{F}_{T}\right),
$$

such that

$$
\int_{F} \lambda_{T}=\int_{F} \lambda_{T^{\prime}} \quad \text { for } F=T \cap T^{\prime} .
$$

Define the average $\tilde{\lambda} \in P^{p}(\mathcal{F})$ as

$$
\tilde{\lambda}=\frac{\sum_{T: F \subset T} \lambda_{T \mid F}}{\sum_{T: F \subset T} 1} .
$$

We have to prove continuity of the averaging operator, i.e.

$$
\|\tilde{\lambda}\|_{S}^{2} \leq c(p) \sum_{T \in \mathcal{T}}\left\|\lambda_{T}\right\|_{S, T}^{2}
$$

where $\left\|\lambda_{T}\right\|_{S, T}^{2}:=\inf _{u \in P^{p}(T)}\left\{\|\nabla u\|_{L_{2}(T)}^{2}+\|u-\lambda\|_{j, \partial T}^{2}\right\}$.

We use $\int_{F} \tilde{\lambda}=\int_{F} \lambda_{T}$ to apply Theorem 3 for estimating

$$
\begin{aligned}
\|\tilde{\lambda}\|_{S}^{2} & =\sum_{T \in \mathcal{T}}\left\|\tilde{\lambda}_{\mid \partial T}\right\|_{S, T}^{2} \preceq \sum_{T \in \mathcal{T}}\left\{\left\|\lambda_{T}\right\|_{S, T}^{2}+\left\|\tilde{\lambda}_{\mid \partial T}-\lambda_{T}\right\|_{S, T}^{2}\right\} \\
& \leq \sum_{T \in \mathcal{T}}\left\{\left\|\lambda_{T}\right\|_{S, T}^{2}+\sum_{F \in \mathcal{F}_{T}}\left\|\tilde{\lambda}_{\partial T}-\lambda_{T}\right\|_{F, 0}^{2}\right\} \\
& (\log p)^{\gamma} \sum_{T \in \mathcal{T}}\left\{\left\|\lambda_{T}\right\|_{S, T}^{2}+\sum_{F \in \mathcal{F}_{T}}\left\|\tilde{\lambda}_{\partial T}-\lambda_{T}\right\|_{F}^{2}\right\} \\
& \preceq(\log p)^{\gamma} \sum_{T \in \mathcal{T}}\left\{\left\|\lambda_{T}\right\|_{S, T}^{2}+\sum_{F \in \mathcal{F}_{T}}\left\|\lambda_{T}\right\|_{F}^{2}\right\} \\
& \preceq(\log p)^{\gamma} \sum_{T \in \mathcal{T}}\left\|\lambda_{T}\right\|_{S, T}^{2}
\end{aligned}
$$

The condition number $\kappa\left(C_{B D D C}^{-1} A\right)$ is given by the continuity bound $c(p)=(\log p)^{\gamma}$. 


\section{Traces and polynomial extensions on the interval}

In this section we collect some properties of Jacobi polynomials which can be found in [42], Chapter 4 , or [1], then we prove trace and extension estimates on the interval. Let $w=$ $(1-x)^{\alpha}(1+x)^{\beta}$ be the weight function, for us $\alpha, \beta \in \mathbb{N}_{0}$ is sufficient. The $n^{t h}$-order Jacobi polynomial $P_{n}^{(\alpha, \beta)}$ is defined by Rodrigues' Formula as

$$
P_{n}^{(\alpha, \beta)}(x):=\frac{1}{(-2)^{n} n ! w(x)} \frac{d^{n}}{d x^{n}}\left(w(x)\left(1-x^{2}\right)^{n}\right) .
$$

There holds the orthogonality relation

$$
\int_{-1}^{1} w P_{n}^{(\alpha, \beta)} P_{m}^{(\alpha, \beta)} d x=\delta_{n, m} \frac{2^{\alpha+\beta+1}}{2 n+\alpha+\beta+1} \frac{(n+\alpha) !(n+\beta) !}{n !(n+\alpha+\beta) !}
$$

and boundary values are

$$
P_{n}^{(\alpha, \beta)}(1)=\left(\begin{array}{c}
n+\alpha \\
n
\end{array}\right)
$$

The Legendre polynomials are $P_{n}:=P_{n}^{(0,0)}$, and the integrated Legendre polynomials are defined as

$$
L_{n}(x)=\int_{-1}^{x} P_{n-1}(s) d s .
$$

We often use

$$
\left\|P_{n}\right\|_{L_{2}([-1,1])}^{2}=\frac{2}{2 n+1}
$$

and we need

$$
(2 n+1) L_{n+1}=P_{n+1}-P_{n-1} .
$$

Parameters can be shifted by

$$
(2 n+\alpha+\beta) P_{n}^{(\alpha-1, \beta)}=(n+\alpha+\beta) P_{n}^{(\alpha, \beta)}-(n+\beta) P_{n-1}^{(\alpha, \beta)},
$$

and by telescoping one obtains for the particular choice $\alpha=1$

$$
(m+\beta+1) P_{m}^{(1, \beta)}=\sum_{n=0}^{m}(2 n+\beta+1) P_{n}^{(0, \beta)} .
$$

Differentiating Jacobi polynomials gives

$$
\frac{d}{d x} P_{n}^{(\alpha, \beta)}=\frac{1}{2}(n+\alpha+\beta+1) P_{n-1}^{(\alpha+1, \beta+1)} .
$$

Lemma 6 (Trace inequality 1D). For $v \in P^{n}$ there holds

$$
v(0)^{2} \leq S(\alpha, \beta, n) \int_{0}^{1} y^{\alpha}(1-y)^{\beta} v(y)^{2} d y
$$

and for every $n$ there exists an $l_{n}^{(\alpha, \beta)} \in P^{n}$ such that $l_{n}^{(\alpha, \beta)}(0)=1$ and

$$
\int_{0}^{1} y^{\alpha}(1-y)^{\beta} l_{n}^{(\alpha, \beta)}(y)^{2} d y \leq S(n, \alpha, \beta)^{-1}
$$


with

$$
S(n, \alpha, \beta)=\frac{(n+\alpha+1) !(n+\alpha+\beta+1) !}{\alpha !(\alpha+1) ! n !(n+\beta) !} .
$$

For a fixed $\alpha$ there holds

$$
S(n, \alpha, \beta) \simeq(n+1)^{\alpha+1}(n+1+\beta)^{\alpha+1} .
$$

Proof. Estimate (7) is sharp for the solution of the constrained minimization problem

$$
\min _{v(0)=1} \int_{0}^{1} y^{\alpha}(1-y)^{\beta} v(y)^{2} d y
$$

By choosing the representation

$$
v(y)=\sum_{k=0}^{n} c_{k} P_{k}^{(\alpha, \beta)}(1-2 y)
$$

the minimization problem can be rephrased as

$$
\min _{\substack{c \in \mathbb{R} n+1 \\ b^{T} c=1}} c^{T} D c
$$

with $b \in \mathbb{R}^{n+1}$ and $D \in \mathbb{R}^{n+1 \times n+1}$ diagonal with components

$$
\begin{aligned}
b_{k} & =P_{k}^{(\alpha, \beta)}(1)=\frac{(k+\alpha) !}{\alpha ! k !} \\
D_{k, k} & =\int_{0}^{1} y^{\alpha}(1-y)^{\beta} P_{k}^{(\alpha, \beta)}(1-2 y)^{2} d y=\int_{-1}^{1}\left(\frac{1-z}{2}\right)^{\alpha}\left(\frac{1+z}{2}\right)^{\beta} P_{k}^{(\alpha, \beta)}(z)^{2} \frac{1}{2} d z \\
& =\frac{1}{2 k+\alpha+\beta+1} \frac{(k+\alpha) !(k+\beta) !}{k !(k+\alpha+\beta) !}
\end{aligned}
$$

Using the method of Lagrange multipliers we obtain

$$
\left(\begin{array}{cc}
D & b \\
b^{T} & 0
\end{array}\right)\left(\begin{array}{l}
c \\
\lambda
\end{array}\right)=\left(\begin{array}{l}
0 \\
1
\end{array}\right)
$$

With the Schur complement

$$
\begin{aligned}
S & =b^{T} D^{-1} b=\sum_{k=0}^{n} \frac{b_{k}^{2}}{D_{k, k}} \\
& =\sum_{k=0}^{n}(2 k+\alpha+\beta+1) \frac{(k+\alpha) !(k+\alpha+\beta) !}{(\alpha !)^{2} k !(k+\beta) !}
\end{aligned}
$$

the solution is given by $\lambda=\frac{-1}{S}$ and $c=\frac{1}{S} D^{-1} b$. The value of the minimum is $S^{-1}$.

By means of the Paule/Schorn implementation [33] of Gosper's algorithm, V. Pillwein computed

$$
S=\frac{(n+\alpha+1) !(n+\alpha+\beta+1) !}{\alpha !(\alpha+1) ! n !(n+\beta) !} .
$$

More on computer algebra techniques in finite element methods is found in [37]. 
We continue with a hand-proof for the assymptotic behavior:

$$
\begin{aligned}
S & \simeq c(\alpha) \sum_{k=0}^{n}(k+1)^{\alpha}(k+\beta+1)^{\alpha+1} \\
& =c(\alpha) \sum_{k=0}^{n}(k+1)^{\alpha} \sum_{j=0}^{\alpha+1}\left(\begin{array}{c}
\alpha+1 \\
j
\end{array}\right)(k+1)^{j} \beta^{\alpha+1-j} \\
& \simeq c(\alpha) \sum_{j=0}^{\alpha+1}\left(\begin{array}{c}
\alpha+1 \\
j
\end{array}\right)(n+1)^{j+\alpha+1} \beta^{\alpha+1-j} \\
& =c(\alpha)(n+1)^{\alpha+1}(n+\beta+1)^{\alpha+1}
\end{aligned}
$$

Lemma 7. For $v \in P^{n}$ there holds

$$
\begin{aligned}
(v(0)-v(1))^{2} & \preceq \log n \int_{0}^{1} y(1-y) v^{\prime}(y)^{2} d y \\
v(0)^{2} & \preceq \log n \int_{0}^{1} y(1-y)\left(v^{\prime}(y)^{2}+v(y)^{2}\right) d y
\end{aligned}
$$

Proof. To verify (8) we follow the lines of Lemma 6 . Now we expand

$$
v(y)=\sum_{k=0}^{n} c_{k} P_{k}^{(0,0)}(1-2 y),
$$

from (6) there follows

$$
v^{\prime}(y)=-\sum_{k=1}^{n} c_{k}(k+1) P_{k-1}^{(1,1)}(1-2 y),
$$

and now

$$
\begin{aligned}
b_{k} & =P_{k}^{(0,0)}(1)-P_{k}^{(0,0)}(-1)=1+(-1)^{k} \\
D_{k, k} & =(k+1)^{2} \int_{0}^{1} y(1-y) P_{k-1}^{(1,1)}(1-2 y)^{2} d y=(k+1)^{2} \frac{k}{(2 k+1)(k+1)},
\end{aligned}
$$

and thus

$$
S=\sum_{k=0}^{p} \frac{b_{k}^{2}}{D_{k, k}}=\sum_{\substack{k=0 \\ k \text { even }}}^{n} \frac{4(2 k+1)}{(k+1) k} \simeq \sum_{k=0}^{n} \frac{1}{k+1} \simeq \log n
$$

Estimate (9) follows from (8) as follows: for $\tilde{v}(y):=(1-y) v(y)$ we apply (8) to obtain

$$
\begin{aligned}
v(0)^{2}=\tilde{v}(0)^{2} & \preceq \log n \int_{0}^{1} y(1-y) \tilde{v}^{\prime}(y)^{2} d y \\
& =\log n \int_{0}^{1} y(1-y)\left[-v(y)+(1-y) v^{\prime}(y)\right]^{2} d y \\
& \preceq \log n \int_{0}^{1} y(1-y)\left(v(y)^{2}+v^{\prime}(y)^{2}\right) d y
\end{aligned}
$$


Next we prove that the minimal energy extension in certain norms is also quasi-optimal in related norms:

Lemma 8. We define for $n, \beta \in \mathbb{N}_{0}$

$$
l_{n}^{\beta}:=\underset{v \in P^{n}, v(0)=1}{\operatorname{argmin}} \int_{0}^{1} y(1-y)^{\beta} v(y)^{2} d y .
$$

Then there holds

$$
\begin{aligned}
\int_{0}^{1} y(1-y)^{\beta} l_{n}^{\beta}(y)^{2} d y & \preceq \frac{1}{(n+1)^{2}(n+\beta+1)^{2}}, \\
\int_{0}^{1}(1-y)^{\beta} l_{n}^{\beta}(y)^{2} d y & \preceq \frac{1}{(n+1)(n+\beta+1)}, \\
\int_{0}^{1} y(1-y)^{\beta+1}\left(\left(l_{n}^{\beta}\right)^{\prime}(y)\right)^{2} d y & \preceq 1 .
\end{aligned}
$$

Proof. The optimizer $l_{n}^{\beta}$ was calculated in the proof of Lemma 6 with $\alpha=1$, namely

$$
l_{n}^{\beta}(y)=\sum_{k=0}^{n} c_{k} P_{k}^{(1, \beta)}(1-2 y),
$$

with $b_{k}=k+1$ and $D_{k, k}=\frac{1}{2 k+\beta+2} \frac{k+1}{k+\beta+1}$. We get

$$
c_{k}=\frac{b_{k}}{D_{k, k} S}=\frac{(2 k+\beta+2)(k+\beta+1)}{S},
$$

and $S \simeq(n+1)^{2}(n+\beta+1)^{2}$. Inequality (10) was proven in Lemma 6 . To verify (11) we utilize (5) to re-expand $l_{n}^{\beta}$ in terms of Jacobi-polynomials $P_{n}^{(0, \beta)}$ :

$$
\begin{aligned}
l_{n}^{\beta}(y) & =\sum_{k=0}^{n} c_{k} \sum_{j=0}^{k} \frac{2 j+\beta+1}{k+\beta+1} P_{j}^{(0, \beta)}(1-2 y) \\
& =\sum_{j=0}^{n} \sum_{k=j}^{n} \frac{2 j+\beta+1}{k+\beta+1} c_{k} P_{j}^{(0, \beta)}(1-2 y) \\
& =\frac{1}{S} \sum_{j=0}^{n} \sum_{k=j}^{n}(2 k+\beta+2)(2 j+\beta+1) P_{j}^{(0, \beta)}(1-2 y) \\
& =\frac{1}{S} \sum_{j=0}^{n}(n+j+\beta+2)(n-j+1)(2 j+\beta+1) P_{j}^{(0, \beta)}(1-2 y)
\end{aligned}
$$

Thus there holds

$$
\begin{aligned}
& \int_{0}^{1}(1-y)^{\beta} l_{n}^{\beta}(y)^{2} d y= \\
& \quad=\sum_{j=0}^{n} \int_{0}^{1}(1-y)^{\beta}\left(P_{j}^{(0, \beta)}(1-2 y)\right)^{2} d y \frac{(n+j+\beta+1)^{2}(n-j+1)^{2}(2 j+\beta+1)^{2}}{S^{2}} \\
& \quad \simeq \sum_{j=0}^{n} \frac{1}{2 j+\beta+1} \frac{(n+j+\beta+1)^{2}(n-j+1)^{2}(2 j+\beta+1)^{2}}{(n+1)^{4}(n+\beta+1)^{4}} \\
& \preceq \frac{1}{(n+1)(n+\beta+1)}
\end{aligned}
$$


From (13) and (6) we get

$$
\left(l_{n}^{\beta}\right)^{\prime}(y)=\frac{1}{S} \sum_{j=1}^{n} \frac{j+\beta+1}{2}(n+j+\beta+2)(n-j+1)(2 j+\beta+1) P_{j-1}^{(1, \beta+1)},
$$

and thus with similar arguments as above

$$
\int y(1-y)^{\beta+1}\left(l_{n}^{\beta}\right)^{\prime}(y)^{2} d y \preceq 1 .
$$

We construct a family of minimal extensions $\left\{e_{i}^{p}: 0 \leq i \leq p\right\}$ similar to Lemma 8 , such that the differences between two consecutive functions is small. We obtain this by weighted averaging of the previously defined $l_{n}^{\beta}$.

Lemma 9. For $i$ such that $p / 2 \leq i \leq p$ we define the weighted average

$$
e_{i}^{p}(y)=\frac{1}{\sum_{k=i}^{p} w_{k}} \sum_{k=i}^{p} w_{k}(1-y)^{k-i} l_{p-k}^{2 k-1}(y) \quad \text { with } w_{k}=(p-k+1)
$$

and for $i<p / 2$ we set

$$
e_{i}^{p}(y):=(1-y)^{\lceil p / 2\rceil-i} e_{\lceil p / 2\rceil}^{p}(y) .
$$

There holds $e_{i}^{p} \in P^{p-i}$, it satisfies the boundary condition $e_{i}^{p}(0)=1$ and the estimates

$$
\begin{aligned}
\int y(1-y)^{2 i-1} e_{i}^{p}(y)^{2} d y & \preceq \frac{1}{p^{2}(p-i+1)^{2}} \\
\int(1-y)^{2 i-1} e_{i}^{p}(y)^{2} d y & \preceq \frac{1}{p(p-i+1)} \\
\int y(1-y)\left(\frac{d}{d y}\left((1-y)^{i} e_{i}^{p}(y)\right)\right)^{2} d y & \preceq 1
\end{aligned}
$$

We define differences of consecutive functions as

$$
d_{i}^{p}(y):=e_{i}^{p}(y)-(1-y) e_{i+1}^{p}(y),
$$

they satisfy $d_{i}^{p} \in P^{p-i}, d_{i}^{p}=0$ for $i<p / 2$, and

$$
\begin{aligned}
\int(1-y)^{2 i-1} d_{i}^{p}(y)^{2} d y & \preceq \frac{i^{2}}{p^{3}(p-i+1)^{3}} \\
\int y(1-y)\left(\frac{d}{d y}\left((1-y)^{i} d_{i}^{p}(y)\right)\right)^{2} d y & \preceq \frac{i^{2}}{p^{2}(p-i+1)^{2}}
\end{aligned}
$$

Proof. We apply the triangle inequality, use (10), and $\sum_{k=i}^{p} w_{k} \simeq(p-i+1)^{2}$ to prove (14):

$$
\begin{aligned}
& \left(\int y(1-y)^{2 i-1} e_{i}^{p}(y)^{2} d y\right)^{1 / 2} \\
& \leq \frac{1}{\sum_{k=i}^{p} w_{k}} \sum_{k=i}^{p} w_{k}\left(\int y(1-y)^{2 i-1}\left((1-y)^{k-i} l_{p-k}^{2 k-1}(y)\right)^{2} d y\right)^{1 / 2} \\
& \preceq \frac{1}{\sum w_{k}} \sum_{k=i}^{p}(p-k+1) \frac{1}{(p-k+1)(p-k+1+2 k-1)} \\
& \preceq \frac{1}{p(p-i+1)}
\end{aligned}
$$


The estimates (15) and (16) follow similarly.

The differences $d_{i}^{p}$ vanish for $i<p / 2$, and (17) is trivially fulfilled for $i<p / 2$. Thus we assume $i \geq p / 2$. We realize that

$$
\begin{aligned}
d_{i}^{p}(y) & =e_{i}^{p}(y)-(1-y) e_{i+1}^{p}(y) \\
= & \frac{1}{\sum_{k=i}^{p} w_{k}} \sum_{k=i}^{p} w_{k}(1-y)^{k-i} l_{p-k}^{2 k-1}(y)-\frac{1}{\sum_{k=i+1}^{p} w_{k}} \sum_{k=i+1}^{p} w_{k}(1-y)^{k-i} l_{p-k}^{2 k-1}(y) \\
= & \frac{w_{i}}{\sum_{k=i}^{p} w_{k}}\left(l_{p-i}^{2 i-1}(y)-(1-y) e_{i+1}^{p}(y)\right) .
\end{aligned}
$$

Since

$$
\frac{w_{i}}{\sum_{k=i}^{p} w_{k}} \simeq \frac{1}{p-i+1}
$$

we get

$$
\begin{aligned}
& \int(1-y)^{2 i-1} d_{i}^{p}(y)^{2} d y \\
& \preceq \frac{1}{(p-i+1)^{2}}\left(\int(1-y)^{2 i-1} l_{p-i}^{2 i-1}(y)^{2} d y+\int(1-y)^{2 i-1} e_{i+1}^{p}(y)^{2} d y\right) \\
& \preceq \frac{1}{(p-i+1)^{3} p}
\end{aligned}
$$

The additional factor $\frac{i^{2}}{p^{2}}$ follows trivially since $p / 2 \leq i \leq p$. Estimate (18) follows similarly.

\section{$5 \quad$ Extension from a vertex}

In this section we define and analyze minimal extensions from a vertex of the reference tetrahedron $T$.

Lemma 10. We define

$$
\tilde{e}_{V}=\underset{v \in P^{p-1}, v(0)=1}{\operatorname{argmin}} \int_{0}^{1} y^{2} v(y)^{2} d y
$$

and

$$
e_{V}(y)=(1-y) \tilde{e}_{V}(y) .
$$

Then $e_{V} \in P^{p}$ with $e_{V}(0)=1$ and $e_{V}(1)=0$, and there holds

$$
\begin{gathered}
\int_{0}^{1} y^{2} e_{V}(y)^{2} d y \preceq p^{-6} \\
\int_{0}^{1} y e_{V}(y)^{2} d y \preceq p^{-4} \\
\int_{0}^{1} y^{2} e_{V}^{\prime}(y)^{2} d y \preceq p^{-2}
\end{gathered}
$$


Proof. With Lemma 6 there follows

$$
\int_{0}^{1} y^{2} e_{V}(y)^{2} d y \leq \int_{0}^{1} y^{2} \tilde{e}_{V}(y)^{2} d y \preceq p^{-6} .
$$

With $\int_{0}^{1} v(y)^{2} \preceq p^{2} \int_{0}^{1} y(1-y) v(y)^{2} d y$, see [39, Thm. 3.96], we get

$$
\int_{0}^{1} y e_{V}(y)^{2} d y=\int_{0}^{1} y(1-y)^{2} \tilde{e}_{V}(y)^{2} d y \preceq p^{2} \int_{0}^{1} y^{2}(1-y)^{3} \tilde{e}_{V}(y)^{2} d y \preceq p^{-4},
$$

and with $\int_{0}^{1} y(1-y) v^{\prime}(y)^{2} d y \preceq p^{2} \int_{0}^{1} v^{2}(y) d y$, which is [39, Thm. 3.95], we get

$$
\begin{aligned}
\int_{0}^{1} y^{2} e_{V}^{\prime}(y)^{2} d y & =\int_{0}^{1} y^{2}\left((1-y) \tilde{e}_{V}^{\prime}(y)-\tilde{e}_{V}(y)\right)^{2} d y \\
& \preceq \int_{0}^{1} y^{2}(1-y)^{2} \tilde{e}_{V}^{\prime}(y)^{2}+\int_{0}^{1} y^{2} \tilde{e}_{V}(y)^{2} d y \\
& \preceq p^{2} \int_{0}^{1} y(1-y) \tilde{e}_{V}(y)^{2} d y+p^{-6} \preceq p^{-2}
\end{aligned}
$$

Theorem 11 (Extension from a vertex). Let $V$ be a vertex of the reference tetrahedron $T$, and $\lambda_{V}$ the corresponding barycentric coordinate. Define the vertex-to-element extension $\mathcal{E}_{V \rightarrow T}$ : $\mathbb{R} \rightarrow P^{p}(T)$ as

$$
\mathcal{E}_{V \rightarrow T} v:=e_{V}\left(1-\lambda_{V}\right) v
$$

Then $u:=\mathcal{E}_{V \rightarrow T} v(V)$ vanishes on the face opposite to $V$ and satisfies

$$
\|\nabla u\|_{L_{2}(T)}^{2}+\sum_{F \in \mathcal{F}_{T}}\|u\|_{j, F}^{2} \preceq\|v\|_{H^{1}(T)}^{2}
$$

Proof. We recall the inverse estimate $|v(V)| \preceq p^{2}\|v\|_{H^{1}(T)}^{2}$. There holds

$$
\left\|\nabla e_{V}\left(1-\lambda_{V}\right)\right\|_{L_{2}(T)}^{2} \simeq \int_{0}^{1}\left(1-\lambda_{V}\right)^{2} e_{V}^{\prime}\left(1-\lambda_{V}\right)^{2} d \lambda_{V} \preceq p^{-2}
$$

and for a face $F$ containing $V$ we have

$$
\left\|e_{V}\right\|_{j, F}^{2} \preceq p^{2}\left\|e_{V}\right\|_{L_{2}(F)}^{2} \simeq p^{2} \int_{0}^{1}\left(1-\lambda_{V}\right) e_{V}\left(1-\lambda_{V}\right)^{2} d \lambda_{V} \preceq p^{-2},
$$

and thus the powers of $p$ cancel out.

\section{Extension from an edge}

In this section we analyze trace operator on edges, and define edge-to-element extension operators. We consider the edge $E=\{(x, 0,0):|x| \leq 1\}$ of the reference tetrahedron $T$ defined by (2). We split the construction into two pieces, one is face-to-element extension, the other one is edge-to-face extension. 
Lemma 12. Define the face-to-element extension $\mathcal{E}_{F \rightarrow T}$ and the element-to-face restriction operator $\mathcal{R}_{T \rightarrow F}$ between the reference tetrahedron $T$ and the reference face $F$ from (2), (3) as

$$
\begin{aligned}
\left(\mathcal{E}_{F \rightarrow T} u\right)(x, y, z) & =u(x, y+z) \\
\left(\mathcal{R}_{T \rightarrow F} w\right)(x, y) & =\int_{0}^{1} w(x, s y,(1-s) z) d s .
\end{aligned}
$$

These operators are mappings between $P^{p}(T)$ and $P^{p}(F)$, preserve the function on the edge $y=z=0$, and are continuous with respect to the norms

$$
\left\|\mathcal{R}_{T \rightarrow F} w\right\|_{L_{2}(F), y} \preceq\|w\|_{L_{2}(T)} \quad\left\|\mathcal{R}_{T \rightarrow F} w\right\|_{H^{1}(F), y} \preceq\|w\|_{H^{1}(T)}
$$

and

$$
\left\|\mathcal{E}_{F \rightarrow T} u\right\|_{L_{2}(T)} \preceq\|u\|_{L_{2}(F), y} \quad\left\|\mathcal{E}_{F \rightarrow T} u\right\|_{H^{1}(T)} \preceq\|u\|_{H^{1}(F), y}
$$

with the norms

$$
\|u\|_{L_{2}(F), y}^{2}:=\int_{F} y u(x, y)^{2} d(x, y) \quad\|u\|_{H^{1}(F), y}^{2}:=\|u\|_{L_{2}(F), y}^{2}+\|\nabla u\|_{L_{2}(F), y}^{2} .
$$

Proof. The proof follows from change of variables via

$$
g:[0,1] \times F \rightarrow T:(s, x, y) \mapsto(x, s y,(1-s) y)
$$

with $\left|\operatorname{det} g^{\prime}\right|=y$, and thus

$$
\int_{T} u(\xi, \eta, \zeta)^{2} d(\xi, \eta, \zeta)=\int_{0}^{1} \int_{F} y u(x, s y,(1-s) y)^{2} d(x, y) d s
$$

Next we study trace and extension operators between the face $F$ and the edge $E$. Continuity is proven with respect to the weighted norm $\|\cdot\|_{H^{1}(F), y}$, and a proper norm on the edge $\|\cdot\|_{E}$.

Expand $u \in P^{p}(F)$ as

$$
u(x, y)=\sum_{i=2}^{p} L_{i}\left(\frac{x}{1-y}\right)(1-y)^{i} u_{i}(y)+x u_{1}(y)-u_{0}(y),
$$

where $u_{i} \in P^{p-i}$. Utilize $L_{i}=\frac{1}{2 i-1}\left(P_{i}-P_{i-2}\right)$, define $u_{i}=0$ for $i>p$, and shift indices

$$
\begin{aligned}
u(x, y) & =\sum_{i=2}^{p} \frac{1}{2 i-1} P_{i}(\cdot)(1-y)^{i} u_{i}(y)-\sum_{i=2}^{p} \frac{1}{2 i-1} P_{i-2}(\cdot)(1-y)^{i} u_{i}(y)+x u_{1}(y)-u_{0}(y) \\
& =\sum_{i=2}^{p} P_{i}(\cdot)(1-y)^{i}\left(\frac{u_{i}}{2 i-1}-\frac{u_{i+2}(1-y)^{2}}{2 i+3}\right)-\frac{1}{3}(1-y)^{2} u_{2}-\frac{1}{5} x(1-y)^{2} u_{3}+x u_{1}(y)-u_{0}(y) \\
& =\sum_{i=0}^{p} P_{i}\left(\frac{x}{1-y}\right)(1-y)^{i}\left(\frac{u_{i}(y)}{2 i-1}-\frac{(1-y)^{2} u_{i+2}(y)}{2 i+3}\right)
\end{aligned}
$$


Thus, $u$ can be re-expanded as

$$
u(x, y)=\sum_{i=0}^{p} P_{i}\left(\frac{x}{1-y}\right)(1-y)^{i} v_{i}(y),
$$

where the $v_{i} \in P^{p-i}$ are given as

$$
v_{i}(y)=\frac{u_{i}(y)}{2 i-1}-\frac{(1-y)^{2} u_{i+2}(y)}{2 i+3} .
$$

Lemma 13. Let $u \in P^{p}(F)$, and $u_{i}, v_{i} \in P^{p-i}$ be the expansion coefficients in (21) and (22). Then there holds

$$
\|u\|_{L_{2}(F), y}^{2} \simeq \sum_{i=0}^{p} \frac{1}{i+1} \int_{0}^{1} y(1-y)^{2 i+1} v_{i}(y)^{2} d y
$$

and

$$
\begin{aligned}
\|\nabla u\|_{L_{2}(F), y}^{2} \simeq & \sum_{i=1}^{p} \frac{1}{i} \int_{0}^{1} y(1-y)^{2 i-1} u_{i}^{2}(y) d y \\
& +\sum_{i=0}^{p} \frac{1}{i+1} \int_{0}^{1} y(1-y)\left(\frac{d}{d y}\left((1-y)^{i} v_{i}(y)\right)\right)^{2} d y
\end{aligned}
$$

Proof. Use the Duffy transform $g:[-1,1] \times[0,1] \rightarrow F:(\xi, y) \mapsto(x, y)=(\xi(1-y), y)$ with $\operatorname{det} g^{\prime}=(1-y)$ to transform the norm

$$
\begin{aligned}
\|u\|_{L_{2}(F), y}^{2} & =\int_{F} y u(x, y)^{2} d(x, y)=\int_{0}^{1} \int_{-1}^{1} y \operatorname{det} g^{\prime} u(\xi, y)^{2} d \xi d y \\
& =\int_{0}^{1} \int_{-1}^{1} y(1-y)\left(\sum_{i=0}^{p} P_{i}(\xi)(1-y)^{i} v_{i}(y)\right)^{2} d \xi d y \\
& =\sum_{i=0}^{p} \int_{-1}^{1} P_{i}(\xi)^{2} d \xi \int_{0}^{1} y(1-y)^{2 i+1} v_{i}(y)^{2} d y
\end{aligned}
$$

To transform the gradient-norm we calculate

$$
\left(g^{\prime}\right)^{-T}=\left(\begin{array}{cc}
\frac{1}{1-y} & 0 \\
\frac{\xi}{1-y} & 1
\end{array}\right)
$$

and note that

$$
\left|\left(g^{\prime}\right)^{-T} v\right|^{2} \simeq(1-y)^{-2} v_{1}^{2}+v_{2}^{2} \quad \forall v \in \mathbb{R}^{2}
$$

Then we get

$$
\begin{aligned}
\|\nabla u\|_{L_{2}(F), y}^{2} & =\int_{0}^{1} \int_{-1+y}^{1-y} y\left|\nabla_{(x, y)} u\right|^{2} d x d y \\
& =\int_{0}^{1} \int_{-1}^{1} y \operatorname{det} g^{\prime}\left|\left(g^{\prime}\right)^{-T} \nabla_{(\xi, y)} u\right|^{2} d \xi d y \\
& \simeq \int_{0}^{1} \int_{-1}^{1} y(1-y)^{-1}\left|\frac{\partial u}{\partial \xi}(\xi, y)\right|^{2} d \xi d y+\int_{0}^{1} \int_{-1}^{1} y(1-y)\left|\frac{\partial u}{\partial y}(\xi, y)\right|^{2} d \xi d y .
\end{aligned}
$$


For the first term we use representation (21):

$$
\begin{aligned}
& \int_{0}^{1} \int_{-1}^{1} y(1-y)^{-1}\left(\sum_{i=2}^{p} L_{i}^{\prime}(\xi)(1-y)^{i} u_{i}(y)+u_{1}(y)\right)^{2} d \xi d y \\
= & \int_{0}^{1} \int_{-1}^{1} y(1-y)^{-1}\left(\sum_{i=1}^{p} P_{i-1}(\xi)(1-y)^{i} u_{i}(y)\right)^{2} d \xi d y \\
= & \sum_{i=1}^{p} \int_{-1}^{1} P_{i-1}(\xi)^{2} d \xi \int_{0}^{1} y(1-y)^{2 i-1} u_{i}(y)^{2} d y
\end{aligned}
$$

For the second term we use representation (22):

$$
\begin{aligned}
& \int_{0}^{1} \int_{-1}^{1} y(1-y)\left(\frac{\partial}{\partial y} \sum_{i=0}^{p} P_{i}(\xi)(1-y)^{i} v_{i}(y)\right)^{2} d \xi d y \\
= & \sum_{i=0}^{p} \int_{-1}^{1} P_{i}(\xi)^{2} d \xi \int_{0}^{1} y(1-y)\left(\frac{d}{d y}\left((1-y)^{i} v_{i}(y)\right)\right)^{2} d y
\end{aligned}
$$

For $u(x)=\sum_{i=2}^{p} u_{i} L_{i}(x)+u_{1} x-u_{0}=\sum_{i=0}^{p} v_{i} P_{i}(x) \in P^{p}(E)$ we define the norm

$$
\|u\|_{E}^{2}:=\sum_{i=1}^{p} \frac{u_{i}^{2}}{i p^{2}(p-i+1)^{2}}+\sum_{i=0}^{p} \frac{v_{i}^{2}}{i+1}
$$

We note that

$$
\sum_{i=0}^{p} \frac{v_{i}^{2}}{i+1} \simeq\|u\|_{L_{2}(E)}^{2} .
$$

Numerical tests indicate that the first sum in (24) is bounded by $\log p\|u\|_{L_{2}(E)}^{2}$, and we decided to keep it in the definition of the norm $\|\cdot\|_{E}$ instead of loosing another log-factor.

Lemma 14 (Trace theorem on edges). Let $u \in P^{p}(F)$. Then there holds

$$
\left\|\left.u\right|_{E}\right\|_{E}^{2} \preceq \log p\|u\|_{H^{1}(F), y}^{2}
$$

Proof. Follows immediately from the definition of $\|\cdot\|_{E}$, trace inequalities Lemma 6 and Lemma 7, and the representation Lemma 13:

$$
\begin{aligned}
\|u\|_{E}^{2}= & \sum_{i=1}^{p} \frac{u_{i}^{2}(0)}{i p^{2}(p-i+1)^{2}}+\sum_{i=0}^{p} \frac{v_{i}^{2}(0)}{i+1} \\
\preceq & \sum_{i=1}^{p} \frac{1}{i p^{2}(p-i+1)^{2}} p^{2}(p-i+1)^{2} \int_{0}^{1} y(1-y)^{2 i-1} u_{i}(y)^{2} d y \\
& +\sum_{i=0}^{p} \frac{1}{1+i} \log p \int_{0}^{1} y(1-y)\left[\left(\frac{d}{d y}\left((1-y)^{i} v_{i}(y)\right)\right)^{2}+\left((1-y)^{i} v_{i}(y)\right)^{2}\right] d y \\
\preceq & \log p\|u\|_{H^{1}(F), y}^{2}
\end{aligned}
$$


Lemma 15 (Extension from edges). For $u(x)=\sum_{i=2}^{p} u_{i} L_{i}(x)+u_{1} x-u_{0} \in P^{p}(E)$ and the functions $e_{i}^{p}$ from Lemma 9 we define the extension operator as

$$
\left(\mathcal{E}_{E \rightarrow F} u\right)(x, y):=\sum_{i=2}^{p} u_{i} L_{i}\left(\frac{x}{1-y}\right)(1-y)^{i} e_{i}^{p}(y)+u_{1} x e_{1}^{p}(y)-u_{0} e_{0}^{p}(y) .
$$

Then here holds

$$
\left\|\mathcal{E}_{E \rightarrow F} u\right\|_{H^{1}(F), y} \preceq\|u\|_{E} .
$$

Proof. We convert the extended function into the Legendre basis as

$$
\mathcal{E}_{E \rightarrow F} u=\sum_{i=0}^{p} P_{i}\left(\frac{x}{1-y}\right)(1-y)^{i} v_{i}(y)
$$

where $v_{i} \in P^{p-i}$ are

$$
v_{i}(y)=\frac{u_{i} e_{i}^{p}(y)}{2 i-1}-\frac{u_{i+2}(1-y)^{2} e_{i+2}^{p}(y)}{2 i+3}
$$

We rewrite

$$
\begin{aligned}
v_{i}(y) & =\left(\frac{u_{i}}{2 i-1}-\frac{u_{i+2}}{2 i+3}\right) e_{i}^{p}(y)+\left(e_{i}^{p}(y)-(1-y)^{2} e_{i+2}^{p}(y)\right) \frac{u_{i+2}}{2 i+3} \\
& =v_{i}(0) e_{i}^{p}(y)+\frac{u_{i+2}}{2 i+3}\left(d_{i}^{p}(y)+(1-y) d_{i+1}^{p}(y)\right) .
\end{aligned}
$$

Note that there holds $u(x)=\sum_{i=0}^{p} v_{i}(0) P_{i}(x)$.

From Lemma 9 and Lemma 13 there follows

$$
\begin{aligned}
\left\|\mathcal{E}_{E \rightarrow F} u\right\|_{L_{2}(F), y}^{2} & \simeq \sum_{i=0}^{p} \frac{1}{i+1} \int_{0}^{1} y(1-y)^{2 i+1}\left(e_{i}^{p}(y) v_{i}(0)+\left(d_{i}^{p}(y)+(1-y) d_{i+1}^{p}(y)\right) \frac{u_{i+2}}{2 i+3}\right)^{2} d y \\
& \preceq \sum_{i=0}^{p} \frac{v_{i}(0)^{2}}{i+1} \frac{1}{p^{2}(p-i+1)^{2}}+\sum_{i=0}^{p} \frac{u_{i+2}^{2}}{(i+1)^{3}} \frac{i^{2}}{p^{3}(p-i+1)^{3}} \\
& \preceq\|u\|_{E}^{2}
\end{aligned}
$$

and

$$
\begin{array}{rl}
\| \nabla \mathcal{E}_{E} \rightarrow F & u \|_{L_{2}(F), y}^{2}=\sum_{i=1}^{p} \frac{u_{i}^{2}}{i} \int y(1-y)^{2 i-1} e_{i}^{p}(y)^{2} d y \\
& +\sum_{i=0}^{p} \frac{1}{i+1} \int_{0}^{1} y(1-y)\left(\frac{d}{d y}\left((1-y)^{i}\left(e_{i}^{p}(y) v_{0}+\left(d_{i}^{p}(y)+(1-y) d_{i+1}^{p}(y)\right) \frac{u_{i+2}}{2 i+3}\right)\right)\right)^{2} d y \\
\preceq & \sum_{i=1}^{p} \frac{u_{i}^{2}}{i} \int y(1-y)^{2 i-1} e_{i}^{p}(y)^{2} d y \\
& +\sum_{i=0}^{p} \frac{v_{i}^{2}}{i+1} \int y(1-y)\left(\frac{d}{d y}\left((1-y)^{i} e_{i}^{p}(y)\right)\right)^{2} d y \\
& +\sum_{i=0}^{p} \frac{u_{i+2}^{2}}{(i+1)^{3}} \int y(1-y)\left(\frac{d}{d y}\left((1-y)^{i}\left(d_{i}^{p}(y)+(1-y) d_{i+1}^{p}(y)\right)\right)^{2} d y .\right.
\end{array}
$$


Now we apply Lemma 9 to estimate

$$
\|\nabla u\|_{L_{2}(F), y}^{2} \preceq \sum_{i=1}^{p} \frac{u_{i}^{2}}{i} \frac{1}{p^{2}(p-i+1)^{2}}+\sum_{i=0}^{p} \frac{v_{i}^{2}(y)}{i+1} \simeq\|u\|_{E}^{2} .
$$

Next we estimate the contributions from the jump - norms. For this, we prove a face-toedge trace lemma in weighted $L_{2}$-norms:

Lemma 16. Let $D=\{(y, z): y \geq 0, z \geq 0, y+z \leq 1\}$. For $v \in P^{n}(D)$ there holds

$$
\int_{0}^{1} y^{\alpha}(1-y)^{\beta} v(y, 0)^{2} d y \preceq(n+1)(n+\alpha+\beta+1) \int_{D} y^{\alpha}(1-y-z)^{\beta} v(y, z)^{2} d(y, z)
$$

Proof. We expand

$$
v(y, z)=\sum_{j=0}^{n} P_{j}^{(\alpha, \beta)}\left(2 \frac{y}{1-z}-1\right)(1-z)^{j} v_{j}(z)
$$

with $v_{j} \in P^{n-j}$, and calculate

$$
\int_{0}^{1} y^{\alpha}(1-y)^{\beta} v(y, 0)^{2} d y=\sum_{j=0}^{n} \int_{0}^{1} y^{\alpha}(1-y)^{\beta} P_{j}^{(\alpha, \beta)}(2 y-1)^{2} d y v_{j}(0)^{2},
$$

and with the change of variables $(y, z)=(\eta(1-z), z)$

$$
\begin{aligned}
& \int_{D} y^{\alpha}(1-y-z)^{\beta} v(y, z)^{2} d(y, z) \\
& \quad=\int_{0}^{1} \int_{0}^{1} \eta^{\alpha}(1-\eta)^{\beta}(1-z)^{\alpha+\beta+1} v(\eta(1-z), z)^{2} d \eta d z \\
& \quad=\sum_{j=0}^{p} \int_{0}^{1} \eta^{\alpha}(1-\eta)^{\alpha} P_{j}^{(\alpha, \beta)}(2 \eta-1)^{2} d \eta \int_{0}^{1}(1-z)^{\alpha+\beta+1+2 j} v_{j}(z)^{2} d z
\end{aligned}
$$

The estimate follows with Lemma 6, i.e.

$$
v_{j}(0)^{2} \preceq(n-j+1)(n-j+\alpha+\beta+1+2 j) \int_{0}^{1}(1-z)^{\alpha+\beta+1+2 j} v_{j}(z)^{2} d z,
$$

for $0 \leq j \leq n$.

Lemma 17. For $u \in P^{p}(E)$ there holds

$$
\left\|\mathcal{E}_{E \rightarrow F} u\right\|_{j, F} \preceq\|u\|_{E}
$$

Proof. By characterization (1) we have to prove the estimate

$$
\left(\mathcal{E}_{E \rightarrow F} u, \sigma\right)_{L_{2}(F)} \preceq\|u\|_{E}\|\sigma\|_{L_{2}(T)} \quad \forall u \in P^{p}(E), \forall \sigma \in P^{p}(T)
$$

We recall

$$
\mathcal{E}_{E \rightarrow F} u=\sum_{i=0}^{p} v_{i}(y) P_{i}\left(\frac{x}{1-y}\right)(1-y)^{i}
$$


with $(25)$

$$
v_{i}(y)=v_{i}(0) e_{i}^{p}(y)+\frac{u_{i+2}}{2 i+3}\left(d_{i}^{p}(y)+(1-y) d_{i+1}^{p}\right)
$$

We expand $\sigma$ as

$$
\sigma=\sum_{i=0}^{p} P_{i}\left(\frac{x}{1-y-z}\right)(1-y-z)^{i} \sigma_{i}(y, z)
$$

with $\sigma_{i} \in P^{p-i}(D)$. By the change of variables $(x, y, z)=(\xi(1-y-z), y, z)$ we have

$$
\begin{aligned}
\|\sigma\|_{L_{2}(T)}^{2} & =\int_{D} \int_{-1+y+z}^{1-y-z} \sigma(x, y, z)^{2} d x d(y, z) \\
& =\int_{D} \int_{-1}^{1}(1-y-z) \sigma(\xi(1-y-z), y, z)^{2} d \xi d(y, z) \\
& =\sum_{i}\left\|P_{i}\right\|_{0}^{2} \int_{D}(1-y-z)^{2 i+1} \sigma_{i}(y, z)^{2} d(y, z) .
\end{aligned}
$$

We expand the inner product, use Lemma 9 and Lemma 16 to estimate

$$
\begin{aligned}
\left(\mathcal{E}_{E \rightarrow F} u, \sigma\right)_{L_{2}(F)}=\int_{0}^{1} \int_{-1+y}^{1-y}\left(\sum_{i=0}^{p} P_{i}\left(\frac{x}{1-y}\right)(1-y)^{i} v_{i}(y)\right)\left(\sum_{j=0}^{p} P_{i}\left(\frac{x}{1-y}\right)(1-y)^{i} \sigma_{i}(y, 0)\right) d x d y \\
=\sum_{i=0}^{p}\left\|P_{i}\right\|^{2} \int_{0}^{1}(1-y)^{2 i+1} v_{i}(y) \sigma_{i}(y, 0) d y \\
\leq \sum_{i=0}^{p}\left\|P_{i}\right\|^{2}\left(\int_{0}^{1}(1-y)^{2 i+1} v_{i}(y)^{2} d y\right)^{1 / 2}\left(\int_{0}^{1}(1-y)^{2 i+1} \sigma_{i}(y, 0)^{2} d y\right)^{1 / 2} \\
\preceq \sum_{i=0}^{p}\left\|P_{i}\right\|^{2}\left(\frac{v_{i}(0)^{2}}{p(p-i+1)}+\frac{u_{i}^{2}}{p^{3}(p-i+1)^{3}}\right)^{1 / 2}\left((p-i+1) p \int_{D}(1-y-z)^{2 i+1} \sigma_{i}(y, z)^{2} d(y, z)\right)^{1 / 2} \\
\leq\left(\sum_{i=0}^{p}\left\|P_{i}\right\|^{2}\left(v_{i}(0)^{2}+\frac{u_{i}^{2}}{p^{2}(p-i+1)^{2}}\right)\right)^{1 / 2}\left(\sum_{i=0}^{p}\left\|P_{i}\right\|^{2} \int_{D}(1-y-z)^{2 i+1} \sigma_{i}(y, z)^{2} d(y, z)\right)^{2} \\
\simeq\|u\|_{E}\|\sigma\|_{L_{2}(T)}
\end{aligned}
$$

Finally we define the edge to element extension $\mathcal{E}_{E \rightarrow T}: P^{p}(E) \rightarrow P^{p}(T)$ as

$$
\mathcal{E}_{E \rightarrow T}:=\mathcal{E}_{F \rightarrow T} \mathcal{E}_{E \rightarrow F}
$$

Theorem 18. For $v \in P^{p}(T)$ define

$$
u:=\left.\mathcal{E}_{E \rightarrow T} v\right|_{E} .
$$

Then $\left.u\right|_{E}=\left.v\right|_{E}$ and there holds

$$
\|u\|_{H^{1}(T)}^{2}+\sum_{F: E \subset F}\|u\|_{j, F}^{2} \preceq \log p\|v\|_{H^{1}(T)}^{2} .
$$

If in addition $v$ vanishes at the end-points of the edge $E$, then $u$ vanishes on faces not containing $E$, and there holds

$$
\|u\|_{H^{1}(T)}^{2}+\|u\|_{j, \partial T}^{2} \preceq \log p\|v\|_{H^{1}(T)}^{2} .
$$

Proof. Follows from the construction of $\mathcal{E}_{F \rightarrow T}$ and $\mathcal{E}_{E \rightarrow F}$, and Lemmas 12, 14, 15, and 17 . 


\begin{tabular}{ccccccc} 
pol deg & $\begin{array}{c}\text { BR - facet } \\
\alpha=5\end{array}$ & $\begin{array}{c}\text { BR - element } \\
\alpha=1.5\end{array}$ & $\begin{array}{c}L_{2} \text {-stab } \\
\alpha=5\end{array}$ & $\begin{array}{c}L_{2} \text {-stab } \\
\alpha=10\end{array}$ & $\begin{array}{c}L_{2} \text {-stab } \\
\alpha=20\end{array}$ & $\begin{array}{c}L_{2} \text {-stab } \\
\alpha=40\end{array}$ \\
\hline 2 & 24.91 & 10.62 & 12.91 & 23.74 & 45.50 & 88.96 \\
4 & 41.41 & 18.64 & 23.62 & 41.19 & 75.63 & 144.65 \\
8 & 59.44 & 33.16 & 42.27 & 67.20 & 116.47 & 214.49 \\
16 & 80.70 & 54.78 & 65.97 & 94.73 & 152.47 & 268.62
\end{tabular}

Table 1: Condition numbers of the BDDC-preconditioned system depending on $p$ and the stabilization method

\section{Numerical results}

In this section we give some computational results for different versions of stabilization terms. The first one is the facet-wise Bassi-Rebay stabilization as we have analyzed. The second one is an element-wise Bassi-Rebay stabilization where

$$
\|u-\lambda\|_{j, \partial T}:=\sup _{\sigma \in\left[P^{p}(T)\right]^{3}} \frac{\int_{\partial T}(u-\lambda) \sigma_{n} d s}{\|\sigma\|_{L_{2}(T)}} .
$$

Here it is enough to choose the stabilization factor $\alpha>1$. The norm is equivalent to the analyzed one (the proof is at some point tricky, and not given here). The developed theory carrys over. The third one is weighted $L_{2}$-stabilization with

$$
\|u-\lambda\|_{j}^{2}:=\alpha \frac{p^{2}}{h}\|u-\lambda\|_{L_{2}(\partial T)}^{2}
$$

Here, the choice of a sufficiently large $\alpha$ is not trivial.

We have chosen $\Omega=(0,1)^{3}$, and used Netgen to generated an unstructured mesh consisting of 184 tetrahedal elements. The condition numbers using a BDDC - preconditioner are given in Table 7. Choosing $\alpha<3$ for the method with $L_{2}$-stabilization does not lead to a coercive discrete problem.

It is clearly seen that the condition number depends on the stabilization term, and it is an advantage of having a method for which small stabilization factors are guaranteed to be stable. As we have proven, the condition numbers show a poly-logarithmic growth for the BR-facet method. It is left the reader to interpret the numbers for $L_{2}$-stabilization, from our analysis there follows only $\kappa \preceq p(\log p)^{\gamma}$ due to norm equivalence (4).

\section{References}

[1] M. Abramowitz and I. A. Stegun. Handbook of mathematical functions. John Wiley \& Sons, 1993.

[2] M. Ainsworth. A preconditioner based on domain decomposition for $h-p$ finite element approximation on quasi-uniform meshes. SIAM J. Numer. Anal., 33:1358-1376, 1996.

[3] P. F. Antonietti and P. Houston. A Class of Domain Decomposition Preconditioners for hp-Discontinuous Galerkin Finite Element Methods. J. Sci. Comput. 46:124-149, 2011.

[4] D. N. Arnold, F. Brezzi, B. Cockburn, and D. Marini. Unified analysis of discontinuous Galerkin methods for elliptic problems. SIAM J. Numer. Anal. 39(5), 1749-1779 (2002). 
[5] I. Babuška, A. W. Craig, J. Mandel, and J. Pitkäranta. Efficient preconditioning for the $p$ version of the finite element method in $R^{2}$. SIAM J. Numer. Anal., 28:624-661, 1991.

[6] F. Bassi and S. Rebay. High-order accurate discontinuous finite element solution of the 2D Euler equations. J. Comp. Phys. 138:251-285, 1997

[7] S. Beuchler, R. Schneider, and Ch. Schwab. Multiresolution weighted norm equivalences and applications. Numer. Math., 98:67-97, 2004.

[8] I. Bică. Iterative substructuring algorithms for the $p$-version finite element method for elliptic problems. PhD-thesis, Couran Institute of Mathematical Sciences, New York University

[9] M. Casarin. Quasi-optimal Schwarz methods for the conforming spectral element discretization. SIAM J. Numer. Anal., 34:2482-2502, 1997.

[10] B. Cockburn, J. Gopalakrishnan, and R. Lazarov. Unified hybridization of discontinuous Galerkin, mixed, and continuous Galerkin methods for second order elliptic problems. SIAM J. Numer. Anal., 47:1319-1365, 2009.

[11] B. Cockburn, G. Kanschat, and D. Schötzau. A note on discontinuous Galerkin divergence-free solutions of the Navier-Stokes equations. J. Sci. Comput., 31:61-73, 2007.

[12] B. Cockburn, G. E. Karniadakis, and C. W. Shu. Discontinuous Galerkin Methods: Theory, Computation and Applications. Springer, 2000.

[13] Demkowicz, L. Computing with hp-adaptive finite elements. Vol. 1. One and two dimensional elliptic and Maxwell problems. Chapman \& Hall/CRC, 2007

[14] C. R. Dohrmann. A preconditioner for substructuring based on constrained energy minimization. SIAM J. Sci. Comput., 25(1):246-258, 2003

[15] M. Dryja and O. B. Widlund. Towards a unified theory of domain decomposition algorithms for elliptic problems. In T. F. Chan, R. Glowinski, J. Périaux, and O. B. Widlund, editors, Third International Symposium on Domain Decomposition Methods for Partial Differential Equations, pages 3-21, Philadelphia, 1990. SIAM.

[16] M. Dubiner. Spectral methods on triangles and other domains. J. Sci. Comput., 6(4), 345-390 (1991).

[17] J. Gopalakrishnan and G. Kanschat. A multilevel discontinuous Galerkin method. Numer. Math. 95(3):527-550, 2003.

[18] B. Guo and W. Cao. An additive Schwarz method for the $h-p$ version of the finite element method in three dimensions. SIAM J. Numer. Anal., 35:632-654, 1998.

[19] M. Griebel and P. Oswald. On the abstract theory of additive and multiplicative Schwarz algorithms. Numer. Math., 70:163-180, 1995.

[20] G. Haase, U. Langer, and A. Meyer. The Approximate Dirichlet Domain Decomposition Method. Part I : An Algebraic Approach, Part II : Applications to 2nd-order elliptic b.v.p. Computing, 47, pp.137-151, pp.153-167, 1991. 
[21] N.Heuer and F. Leydecker. An extension theorem for polynomials on triangles. Calcolo 45(2): 69-85, 2008.

[22] N: Heuer, F. Leydecker, and E. P. Stephan. An interative substructuring method for the hp-version of the BEM on quasi-uniform triangular meshes. Numer. Methods Partial Differential Eq. 23(4): 879-903, 2007.

[23] J. S. Hesthaven and T. Warburton. Nodal Discontinuous Galerkin Methods-Algorithms, Analysis and Applications. Text in Applied Mathematics. Springer, 2007.

[24] M. Juntunen and R. Stenberg. On a mixed Discontinuous Galerkin method. ETNA, $32: 17-32,2008$.

[25] G. E. Karniadakis and S. J. Sherwin. Spectral/hp Element Methods for Computational Fluid Dynamics. Oxford Science Publications, 2005

[26] A. Klawonn, O. B. Widlund, and M. Dryja. Dual-primal FETI methods for threedimensional elliptic problems with heterogeneous coefficients. SIAM J. Numer. Anal. 40(1):159-179, 2002.

[27] V. Korneev and S. Jensen. Domain decomposition preconditioning in the hierarchical p-version of the finite element method. Appl. Numer. Math., 29:479-518, 1999.

[28] V. G. Korneev and U. Langer. Domain Decomposition and Preconditioning, Chapter 19 in Part I of the Encyclopedia of Computational Mechanics, ed. by E. Stein, R. de Borst and Th.J.R. Hughes, John Wiley \& Sons, 44 pages, 2004.

[29] V. G. Korneev, U. Langer, and L. Xanthis. On Fast Domain Decomposition Solving Procedures for hp-Discretizations of 3D Elliptic Problems. Computational Methods in Applied Mathematics, Vol. 3, No. 4, pp. 536-559, 2003.

[30] C. Lehrenfeld. Hybrid Discontinuous Galerkin methods for solving incompressible flow problems. Master Thesis, RWTH Aachen, 2010

[31] J. Li and O. B. Widlund. FETI-DP, BDDC, and block Cholesky methods. Internat. J. Numer. Methods Engrg. 66(2):250-271, 2006

[32] R. Muñoz-Sola. Polynomial liftings on a tetrahedron and applications to the $h p$-version of the finite element method in three dimensions. SIAM J. Numer. Anal., 34(1):282-314, 1997.

[33] P. Paule and M. Schorn. A Mathematica version of Zeilberger's Algorithm for proving binomial coefficient identities. J. Symbolic Comput. 20:673-698, 1995. Software available from www.risc.jku.at/research/combinat/software/PauleSchorn

[34] L. Pavarion. Additive Schwarz methods for the $p$-version finite element method. Numer. Math., 66:493-515, 1994

[35] L. Pavarino. BDDC and FETI-DP preconditioners for spectral element discretizations Comp meth appl mech eng 196:1380-1388, 2007 
[36] L. F. Pavarino and O. B. Widlund. A polylogarithmic bound for an iterative substructuring method for spectral elements in three dimensions. SIAM J. Numer. Anal. 33(4).1303-1335, 1996.

[37] V. Pillwein. Computer Algebra Tools for Special Functions in High Order Finite Element Methods. PhD thesis, Johannes Kepler University Linz, 2008.

[38] J. Schöberl and J. Melenk and C. Pechstein and S. Zaglmayr. Additive Schwarz Preconditioning for p-Version Triangular and Tetrahedral Finite Elements. IMA Journal of Numerical Analysis, 28:1-24,2008.

[39] C. Schwab. p- and hp-Finite Element Methods. Theory and Applications in Solid and Fluid Mechanics. Oxford Science Publications, 1998.

[40] S. J. Sherwin and M. Casarin. Low Energy Basis Preconditioning for Elliptic Substructured Solvers Based on Unstructured Spectral/ hp Element Discretisations. Journal of Computational Physics, 171:394-417, 2001

[41] B. Szabó and I. Babuška. Finite Element Analysis. Wiley, 1991.

[42] G. Szegoö. Orthogonal Polynomials. AMS Colloquium Publications, Vol 23, 1939 (reprinted 2003).

[43] A. Toselli and O. Widlund. Domain Decomposition Methods - Algorithms and Theory. Springer Series in Computational Mathematics, Vol. 34, 2005. 